\title{
Photoacoustic and Transmission Studies of SiC Polytypes
}

\author{
A.C. de Oliveira ${ }^{\mathrm{a} *}$, J.A. Freitas Jr. ${ }^{\mathrm{b}}$, W.J. Moore ${ }^{\mathrm{b}}$, A. Ferreira da Silva ${ }^{\mathrm{c}}$, I. Pepe ${ }^{\mathrm{c}}$, \\ J. Souza de Almeida ${ }^{\mathrm{c}, \mathrm{e}}$, G.C.B. Braga ${ }^{\mathrm{d}}$, J.M. Osório-Guillen ${ }^{\mathrm{e}}$, C. Persson $^{\mathrm{e}}$, R. Ahuja $^{\mathrm{e}}$ \\ anstituto de Física, Universidade de Brasília, 70919-970 Brasília - DF, Brazil \\ ${ }^{\mathrm{b}}$ Naval Research Laboratory, Washington, D.C. 20375, USA \\ 'Instituto de Física, Universidade Federal da Bahia, 40210-340 Salvador - BA, Brazil \\ ${ }^{\mathrm{d}}$ Corpo de Bombeiros Militar do Distrito Federal, Brasília - DF, Brazil \\ ${ }^{\mathrm{e}}$ Department of Physics, Uppsala University, SE-75121, Uppsala, Sweden
}

Received: January 02, 2002; Revised: September 30, 2002

\begin{abstract}
The optical bandgap energies (OBGE) of 3C, 15R, 6H and 4H-SiC have been investigate experimentally by transmission and photoacoustic spectroscopies. The measurements were performed on $470 \mu \mathrm{m}$ thick wafers. The OBGE obtained from both spectroscopies for different polytypes show very good agreement. In order to have a better understanding of these materials calculations of eletronic band structure were performed by the full-potential linearized augmented plane wave (FPLAPW) method. For the OBGE the results are compared to the measurements agreeing closely over the energies of those polytypes.
\end{abstract}

Keywords: SiC Polytypes, optical bandgap energies, plane wave method

\section{Introduction}

In the past decade techniques have been developed for the fabrication of bulk and epitaxial films of various $\mathrm{SiC}$ polytypes $^{1}$. These developments have revived the possibility of $\mathrm{SiC}$ system applications in high temperature, high power, and high-speed electronics devices ${ }^{2,3}$. In this work we will discuss the use of photoacustic spectroscopy, a contactless thermal technique, as well as the transmission, to monitor the optical properties of the $3 \mathrm{C}-\mathrm{SiC}, 4 \mathrm{H}-\mathrm{SiC}$, $6 \mathrm{H}-\mathrm{SiC}$ and $15 \mathrm{R}-\mathrm{SiC}$ polytypes.

Photothermal spectroscopy has achieved great developments in the last two decades, and it has been successfully applied to semiconductors samples in case of powders, nonpolished samples, crystalline, polycrystalline or amorphous thin films, multilayered structure, etc. One of the principal advantage of photoacoustic spectroscopy over other optical measurements, e.g. optical absorption, is that produce similar spectra, and may provide direct information of the nonradiative recombination process and, consequently, compliments the absorption and the photoluminescence spectroscopy. In the present work we will investigate the bandgap energies both experimentally and theoretically.
Theoretically by the full potential linearized augmented plane wave (FPLAPW) method.

\section{Experimental Technique}

Photoacoustic spectroscopy (PAS) is directly related to the periodic heat generated in a sample due to the absorption of modulated light. Generally the PAS experimental set up consists of a sample enclosed in a sealed cell at atmospheric pressure coupled to a sensitive microphone. Therefore the temperature of the encapsulated gas is modulated at the excitation frequency and the microphone detects the resulting dynamic pressure. The signal generated by this process depends on the amount of heat generated in the sample due to its optical absorption coefficient, the nonradioactive processes efficiency, and the thermal diffusivity. The study of the dependence of the sample absorption coefficient on the light source wavelength allows one to determine optical and electronic properties of opaque and transparent materials ${ }^{4}$.

In order to minimize the background noise a new designed photoacoustic cell with, double walls was built. This new device consists of two concentric cylindrical wall cell

*e-mail: aderbal@unb.br 


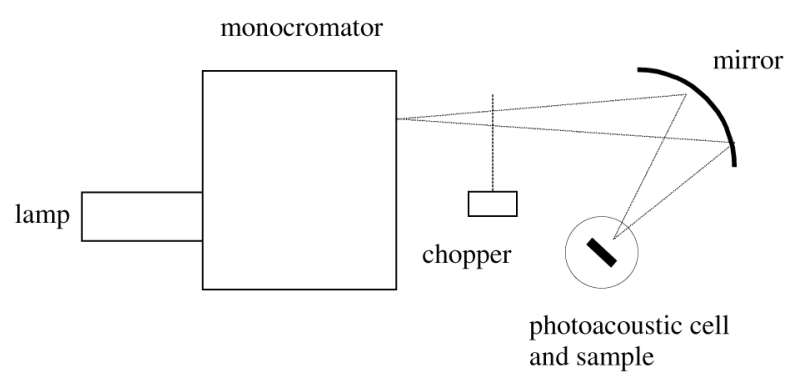

Figure 1. Schematic Experimental Setup.

with uv-quartz windows. The space between the walls was filled with air at atmospheric pressure. The internal cell cavity was gold plated in order to minimize unwanted radiation absorption. Air was used as coupling media between the sample and the microphone. A 1/2-inch condenser microphone (Bruel and Kjær, model 3547) was used to monitor the change in the pressure inside the cell. A 150 Watts Xe lamp, with a ellipsoidal reflector, couple to a $0.22 \mathrm{~m}$ double monocromator (Spex model 1680) was used as variable wavelength light source. The light was chopped at a frequency of $5 \mathrm{~Hz}$, to improve the signal/noise ratio. The system was computer controlled. The schematic of the experimental setup is shown in Fig. 1. All the PAS spectra of the samples were normalized to the spectra of a highly absorbing film. PAS has been used to measure the bandgap energy of the polytypes.

The transmission measurements were performed placing the sample, attached to a holder with a $1 \mathrm{~mm} \times 2 \mathrm{~mm}$ aperture, in front of the exit slit of the variable wavelength light source used in the photoacustic experiments. The intensity of the transmitted light was monitored with a Si-photodiode. All the sample transmission spectra were normalized to the spectra of the light transmitted through the sample holder without the sample. The transmission experiment was carried out in the same set of sample studied by PAS.

\section{Computation Method}

The computational method, for the calculation of the bandgap, full potential linearized augmented plane wave (FPLAPW) method within the density-functional theory and local-density approximation (LDA) and using the correlation potential of Perdew and Wang $(\mathrm{PW})^{5}$. The LDA is known to underestimate the bandgap energy Eg for semiconductors ${ }^{6}$.

Because of the incorrect bandgap, one cannot guarantee that the calculations yield an accurate dielectric function. However, it has been shown by Del Sole and Girlanda ${ }^{7}$ that the LDA combined with the scissors-operator approximation describes the optical spectrum rather well. We have therefore made an estimate of the correction $\Delta_{\mathrm{g}}$ to the bandgap by using a quasi-particle method proposed by Bechstedt and Del Solo ${ }^{8}$. Their model for the correction is based on the difference in self-energies obtained from the LDA and the $\mathrm{W}$ approximation. The correction is given by ${ }^{6,8}$

$$
\Delta_{g}=\frac{e^{2} q_{T F}}{2 \pi \varepsilon(0)} \int_{0}^{\infty} \frac{1}{1-t^{2}}\left(\left(1-\alpha_{p}\right) f\left(q_{T F}^{r} A^{t}\right)+\left(1+\alpha_{p}\right) f\left(q_{T F}{ }^{r} A^{t}\right)\right)^{2} d t
$$

$$
f(x)=\frac{1-10 x^{2} / 3+x^{4}}{\left(1+x^{2}\right)^{6}} ; \quad r_{A}=r_{B}=\frac{a}{4 \pi \cdot 1.7}
$$

In the equation above $q_{T F}$ is the Thomas-Fermi wave number, $\varepsilon(0)$ is the static dielectric function, $\alpha_{p}$ the polarity of the interatomic interaction ${ }^{8}$ calculated to be 7.67 , and $a$ is the lattice constant for each of the polytypes ${ }^{5}$.

We have employed experimental values for the lattice dielectric function. For 3C-(6H-) $\mathrm{SiC} \varepsilon(0)=9.72$ (9.78). The dielectric constant for $6 \mathrm{H}-\mathrm{SiC}$ are the geometric average values of the transverse and longitudinal components: $\varepsilon(0)_{\perp}=9.66$ and $\varepsilon(0)_{\|}=10.03$,

$$
\varepsilon(0)=\frac{2 \varepsilon(0)_{\perp}+\varepsilon(0)_{\|}}{3}
$$

Furthermore, $4 \mathrm{H}-\mathrm{SiC}$ and $15 \mathrm{R}-\mathrm{SiC}$ are presumed to have the same lattice dielectric function as $6 \mathrm{H}-\mathrm{SiC}$.

\section{Experimental Results}

In Figs. 2 and 3, we show the room temperature photoacoustic and transmission spectra respectively. The optical bandgap energies derived from these results are shown

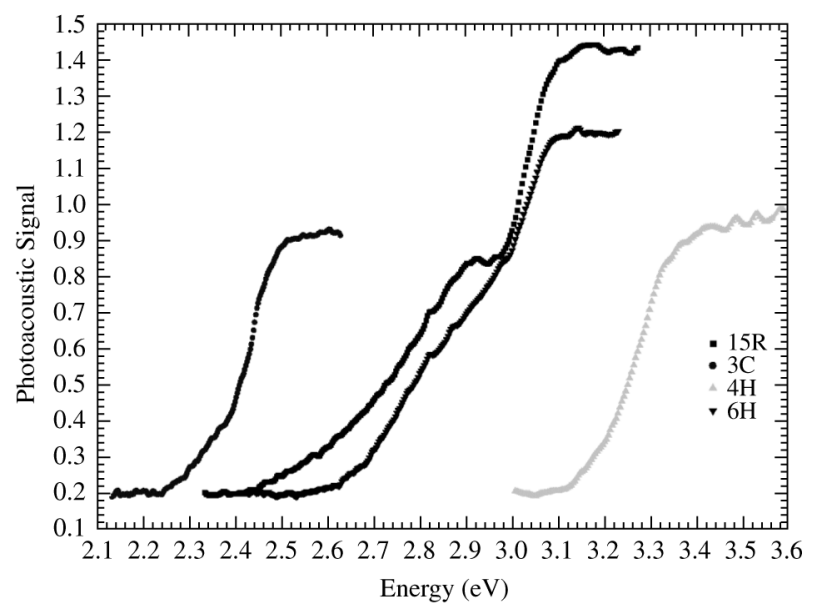

Figure 2. Photoacoustic signals as a function of energy for $\mathrm{SiC}$ polytypes. 
Table 1. Obtained values of the bandgap energies for Photoacoustic (PA) and Transmission (TR) measurements. The calculated $\mathrm{E}_{\mathrm{g}}(\mathrm{C})$ and quasi-particle correction $\Delta_{\mathrm{g}}$ are shown.

\begin{tabular}{lccccc}
\hline Material & $\mathrm{E}_{\mathrm{g}}(\mathrm{PA})(\mathrm{eV})$ & $\mathrm{E}_{\mathrm{g}}(\mathrm{TR})(\mathrm{eV})$ & $\mathrm{E}_{\mathrm{g}}(\mathrm{C})(\mathrm{eV})$ & $\Delta_{\mathrm{g}}(\mathrm{eV})$ & $\left(\mathrm{E}_{\mathrm{g}}(\mathrm{C})+\Delta_{\mathrm{g}}\right)(\mathrm{eV})$ \\
\hline $3 \mathrm{C}-\mathrm{SiC}$ & $2.44 \pm 0.02$ & $2.44 \pm 0.02$ & $1.30 \pm 0.01$ & 1.24 & $2.54 \pm 0.01$ \\
$15 \mathrm{R}-\mathrm{SiC}$ & $3.02 \pm 0.02$ & $3.04 \pm 0.02$ & $1.76 \pm 0.01$ & 1.23 & $2.99 \pm 0.01$ \\
$6 \mathrm{H}-\mathrm{SiC}$ & $3.03 \pm 0.02$ & $3.05 \pm 0.02$ & $1.97 \pm 0.01$ & 1.23 & $3.20 \pm 0.01$ \\
$4 \mathrm{H}-\mathrm{SiC}$ & $3.30 \pm 0.02$ & $3.30 \pm 0.02$ & $2.17 \pm 0.01$ & 1.23 & $3.40 \pm 0.01$ \\
\hline
\end{tabular}

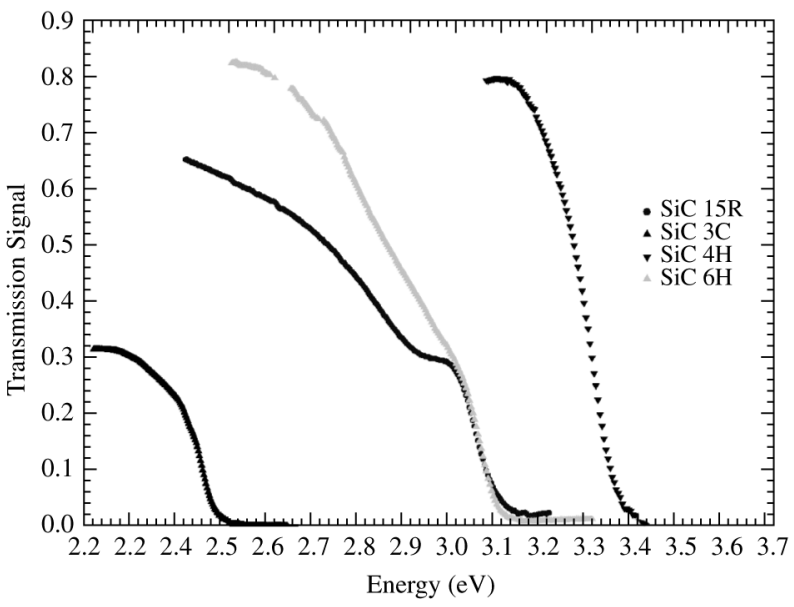

Figure 3. Transmission signal as a function of energy for $\mathrm{SiC}$ polytypes.

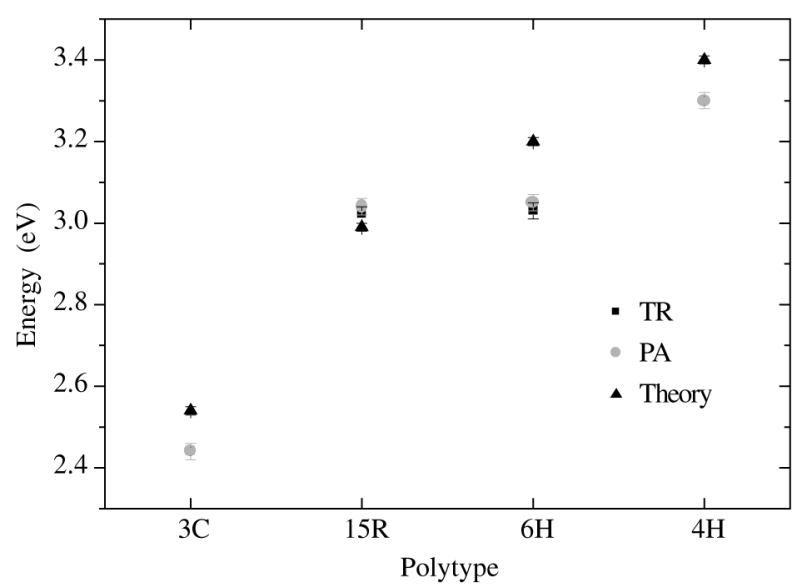

Figure 4. Optical bandgap energies as function of $\mathrm{SiC}$ polytypes. (The error in the energies is less than $0.02 \mathrm{eV}$, so the bars are inside the dots.) in Table 1 together with the calculated values. The results are compared to the measurements agreeing closely over the energies of those polytypes. They are also shown in Fig. 4.

\section{Conclusion}

We have measured the optical bandgap energies of 3C-, 4H-, 6H- and 15R-SiC, using photoacoustic and transmission spectroscopies. The results are in good agreement with our full-potential calculations.

\section{Acknowledgements}

This work was financially supported by the Brazilian agencies CNPq and FAP-DF, Swedish agencies TFR, NFR and SSF, and work at the Naval Research Laboratory by the Office of Naval Research.

\section{Reference}

1. Davis, R.F. Proc. of 5th. SiC and Related Materials Conf., Washington (1993), Inst. Phys. Conf. Ser., v. 137 p. 1, 1994.

2. Morelli, D.; Heremans, J.; Beetz, C.; Woo, W.S.; Harris, G.; Taylor, C. Proc. of 5th. SiC and Related Materials Conf., Washington (1993), Inst. Phys. Conf. Ser., v. 137, p. 313, 1994.

3. Mokoç, H.; Strite, S.; Gao, G.B.; Lin, M.E.; Sverdlov, B.; Burns, M. J. Appl. Phys., v. 76, p. 1363, 1994.

4. Silva, L.A.; de Andrade, J.B.; Moysés, Araújo, C.; Nakamura, D.; da Silva, A.F.; Massunaga, M.S.O.; Vargas, H.; Phys. Chem. Chem. Phys., v. 3, p. 4800, 2001.

5. Persson, C.; Lindefelt, U. Phys. Rev. B, v. 54, p. 10257, 1996; J. Appl. Phys., v. 82, p. 5496, 1997.

6. Persson, C.; da Silva, A.F.; Ahuya, R.; Johansson, B. J. Cryst. Growth, v. 235, p. 397, 2001; J. Phys.: Condens. Matter, v. 13, p. 8945, 2001.

7. Del Sole, R.; Girlanda, R. Phys Rev B, v.48, p. 11789, 1993. 8. Bechstedt, F.; Del Sole, R. Phys. Rev B, v. 38, p. 7710, 1998. 